\title{
Applicability of NRTL Model for Prediction of the Viscosity of Alkanolamine + Water Mixtures
}

\author{
Sumudu S. Karunarathne Lars E. Øi \\ Faculty of Technology, Natural Sciences and Maritime Sciences, University of South-Eastern Norway, Norway, \\ \{Sumudu.karunarathne, lars.oi\}@usn. no
}

\begin{abstract}
This study discusses the applicability of the non-random two-liquid (NRTL) model to represent viscosity for MEA (monoethanol amine) $+\mathrm{H}_{2} \mathrm{O}$ and AMP (2-amino2-methyl-1-propanol) + MEA (monoethanol amine) + $\mathrm{H}_{2} \mathrm{O}$ mixtures under different amine concentrations at temperature ranges of $293.15 \mathrm{~K}-363.15 \mathrm{~K}$ and 293.15 $\mathrm{K}-343.15 \mathrm{~K}$ respectively. The NRTL model is adopted to determine excess Gibbs free energy of mixing $\Delta G^{E *}$ and the Eyring's viscosity model based on absolute rate theory is used to obtain excess free energy of activation for viscous flow $\Delta F^{E *}$. The correlations are proposed for $\Delta F^{E *}$ as a function of concentration of the components, temperature and $\Delta G^{E *}$. Correlations are capable of representing measured viscosities at $1.3 \%$ and $0.3 \%$ of absolute average relative deviation (AARD \%) for MEA $+\mathrm{H}_{2} \mathrm{O}$ and $\mathrm{AMP}+\mathrm{MEA}+\mathrm{H}_{2} \mathrm{O}$ mixtures respectively. These deviations are acceptable for engineering calculations and correlations can be used in process design and simulations like Aspen HYSYS and ASPEN Plus.
\end{abstract}

Keywords: $\quad$ NRTL model, Eyring's viscosity model, $M E A, A M P$

\section{Introduction}

In the design of units involving liquid flow like gas/liquid separators and heat exchangers, it is important to predict reasonably accurate physical properties like viscosity. Correlations depending on parameters from experiments are available for some systems. Estimation methods without the need for fitted parameters is a possibility. A possibility to use parameters from e.g. vapor/liquid equilibrium models to predict viscosity.

In post combustion $\mathrm{CO}_{2}$ capture, the physical properties of aqueous alkanolamine solutions is a key factor in various aspects such as equipment design, modeling and simulations of absorber and desorber columns. Physical properties are present in various mass and heat transfer correlations and interfacial area correlations that are necessary to evaluate in engineering applications. Accordingly, the viscosity data of aqueous alkanolamine mixtures are highly relevant to build correlations to predict viscosities for unmeasured conditions. Further correlations developed for the viscosity of aqueous alkanolamines can be used to develop correlations for the viscosity of $\mathrm{CO}_{2}$ loaded alkanolamine mixtures.

Correlations based on statistical regression for the viscosity data have high uncertainties beyond the experimental range. The approach of Redlich-Kister (Redlich and Kister, 1948) type polynomial to fit physical properties is widely used and Islam et al., (2004) and Hartono et al., (2014) have taken this approach for viscosity data of aqueous MEA solutions. The Grunberg and Nissan model was used by Mandal $e t$ al., (2003) to correlate different aqueous tertiary mixtures. The McAllister model (McAllister, 1960) based on Eyring's absolute rate theory for dynamic viscosity (Eyring, 1936) is used by Amundsen et al., (2009) and Lee and Lin, (1995) for aqueous MEA solutions and found the parameters to fit measured viscosities. These models are capable of predicting viscosities at acceptable accuracies within the experimental range and can be used in engineering designs.

The thermodynamic information like vapor-liquid equilibrium (VLE) of liquid mixtures can be combined with a viscosity model and such models may be stated as thermodynamics-viscosity models (Cao et al., 1993). The VLE data delivers information about molecular interaction, which can be used in local composition models like nonrandom two-liquid (NRTL) and UNIQUAC. This approach has been applied several times for various multicomponent liquid mixtures. Martins et al., (2000) discussed the applicability of the UNIQUAC model for the viscosity predictions of binary and ternary systems. Novak et al., (2004) discussed segment based Eyring-NRTL viscosity model, which was concerned about the similarities between intermolecular friction and viscosity with a local composition model like NRTL to model excess properties as both are affected by nearest neighbor molecules. The viscosity of electrolyte solutions using Eyrings's absolute rate theory has been discussed to replace excess free energy of activation for viscous flow with Gibbs free energy of mixing (Hu, 2004). For electrolyte solutions of MEA (monoethanol amine) + $\mathrm{H}_{2} \mathrm{O}+\mathrm{CO}_{2}$, the excess free energy of activation for viscous flow was replaced by the Gibbs free energy of mixing that was calculated using the electrolyte-NRTL model (Matin et al., 2013). 
This study investigates the possibility to relate excess Gibbs free energy of mixing with the excess free energy of activation for viscous flow from Eyring's absolute rate theory to predict viscosities at different compositions and temperatures of MEA $+\mathrm{H}_{2} \mathrm{O}$ and AMP (2-amino-2-methyl-1-propanol) + MEA $+\mathrm{H}_{2} \mathrm{O}$ mixtures. Measured density and viscosity data were used to calculate the excess free energy of activation for viscous flow. The NRTL model was adopted for calculating excess Gibbs free energy of mixing and compared with the excess free energy of activation for viscous flow for the considered mixtures. Finally, viscosity predictions were compared with measured data and the accuracy was determined by calculating average absolute relative deviation (AARD \%).

\section{Methodology}

\subsection{Dynamic Viscosity Based on Eyring's Absolute Rate Theory}

A universal model to predict the viscosity of any solution is challenging as solutions exhibit different characteristics that are difficult to discuss in one model. Most of the amine solutions and their blends that are discussed in amine-based $\mathrm{CO}_{2}$ capture shows Newtonian behavior as the molecular weights are less than $5000 \mathrm{~g} \cdot \mathrm{mol}^{-1}$ (Bird et al., 2002). Introducing a qualitative picture of the mechanism of momentum transport of liquids, Eyring and coworkers developed a model to predict the viscosity of liquids from other physical properties (Eyring, 1936; Bird et al., 2002).

Eyring's viscosity model for Newtonian fluids is given in (1) and is valid for both pure liquids and liquid mixtures (Martins et al., 2000).

$$
\eta=\frac{h N}{V} \exp \left(\frac{\Delta F^{*}}{R T}\right)
$$

Where, $\eta, V, \Delta F^{*}, T, h, N$ and $R$ are dynamic viscosity, molar volume, free energy of activation for viscous flow, temperature, Planck's constant, Avogadro's number and the gas constant respectively.

In order to compare with ideal solutions and to calculate the excess free energy of activation properties $\Delta F^{E *}$, following (2) and (3) are obtained by using (1).

$$
\begin{aligned}
& \ln (\eta V)=\ln (\eta V)_{\text {ideal }}+\frac{\Delta F^{E^{*}}}{R T} \\
& \ln (\eta V)=\sum_{i} x_{i} \ln \left(\eta_{i} V_{i}^{o}\right)+\frac{\Delta F^{E^{*}}}{R T}
\end{aligned}
$$

Where, $x_{i}, \eta_{i}, V_{i}^{o}$ and $\Delta F^{E *}$ are mole fraction, viscosity of pure liquids, molar volume of pure liquids and excess free energy of activation for viscous flow respectively.
In this approach, the combination of terms of an ideal mixture and excess energy leads to an expression of viscosity in a real mixture. The ideal term of the (2) is calculated using the properties of pure liquids as given in the (3). The term $\Delta F^{E *} / R T$ describes the non-ideality of the solution viscosity (Matin et al., 2013) and an appropriate model can enhance the prediction of the viscosity. Here, the possibility of using Gibbs free energy of mixing is discussed as it has been related in various ways to $\Delta F^{E *}$ in the literature. Generally, it is related as Gibbs free energy, excess Gibbs energy through proportionality factor, Gibbs free energy of mixing and Gibbs free energy of mixing multiplied by a general constant (Matin et al., 2013). This study investigates the excess Gibbs free energy of mixing for $\mathrm{MEA}+\mathrm{H}_{2} \mathrm{O}$ and AMP + MEA $+\mathrm{H}_{2} \mathrm{O}$ mixtures and compares it with $\Delta F^{E *}$ calculated from the measured density and viscosity data. The NRTL model was adopted to calculate Gibbs free energy of mixing for different compositions and temperatures of the mixtures.

\subsection{NRTL Model}

The local composition theory explains the deviation of local compositions from the bulk composition due to different strength of attractions among the molecules in the mixture. The non-random two liquid model (NRTL) is based on the local composition theory as Wilson's model (Wilson, 1964), which explains the composition variations. For a solution of $m$ components, the excess Gibbs free energy of mixing is given as (Prausnitz et al., 1999)

$$
\begin{aligned}
& \frac{\Delta G^{E *}}{R T}=\sum_{i=1}^{m} x_{i} \frac{\sum_{j=1}^{m} \tau_{j i} G_{j i} x_{j}}{\sum_{l=1}^{m} G_{l i} x_{l}} \\
& \tau_{j i}=\frac{g_{j i}-g_{i i}}{R T} \\
& G_{j i}=\exp \left(-\alpha_{j i} \tau_{j i}\right) \quad\left(\alpha_{j i}=\alpha_{i j}\right)
\end{aligned}
$$

Where, $g_{j i}$ and $g_{i i}$ are energy parameters to characterize $i-j$ and $i-i$ interactions respectively. $\alpha_{j i}$ is a nonrandomness parameter.

Then the $\Delta G_{m i x}^{*}$ is calculated as a sum of both ideal mixing and an excess term due to the non-ideal behavior of the solutions.

A study performed by Schmidt et al., (2007) on VLE and NRTL model for various aqueous amine solutions provide binary interaction parameters for MEA $+\mathrm{H}_{2} \mathrm{O}$ mixtures. A similar work done by Hartono et al., (2013) found relevant parameters for AMP $+\mathrm{H}_{2} \mathrm{O}$ mixtures. There is a lack of information about interaction parameters between AMP and MEA. Hence, for the tertiary AMP + MEA $+\mathrm{H}_{2} \mathrm{O}$ system, parameters from two binary solutions were used for the calculations. It is also possible to use the commercial process simulation program Aspen Plus to perform all the 
Table 1. Summary of the Compositions and Temperatures Considered for the Density and Viscosity Measurements of Aqueous Amine Mixtures.

\begin{tabular}{|c|c|c|c|}
\hline Solution & \multicolumn{2}{|c|}{ Composition / wt \% (by weight) } & Temperature / $K$ \\
\hline $\mathrm{MEA}+\mathrm{H}_{2} \mathrm{O}$ & \multicolumn{2}{|c|}{$0-100(\mathrm{MEA})$} & $293.15-363.15$ \\
\hline \multirow[t]{3}{*}{$\mathrm{AMP}+\mathrm{MEA}+\mathrm{H}_{2} \mathrm{O}$} & $21 / 9 / 70$ & \multirow{3}{*}{$\left(\mathrm{AMP} / \mathrm{MEA} / \mathrm{H}_{2} \mathrm{O}\right)$} & \multirow{3}{*}{$293.15-343.15$} \\
\hline & $24 / 6 / 70$ & & \\
\hline & $27 / 3 / 70$ & & \\
\hline
\end{tabular}

excess free energy of mixing calculations as it has binary interaction parameters for many components in the data banks. For the missing binary interactions parameters of NRTL model, the UNIFAC model can be used to make estimations.

The density and viscosity of mixtures were measured using a DMA 4500 vibrational density meter and Physica MCR 101 rheometer with a double-gap pressure cell XL from Anton Paar. The properties were measured at different compositions and temperatures as given in Table 1 .

\section{Results and Discussion}

The spontaneous mixing of MEA and $\mathrm{H}_{2} \mathrm{O}$ gives negative values for Gibbs free energy of mixing. The excess Gibbs free energy $\Delta G^{E *}$ of mixing was analyzed for the compositions of $x_{M E A}$ from 0 to 1 of MEA $+\mathrm{H}_{2} \mathrm{O}$ mixtures. Figure 1 illustrates the calculated $\Delta G^{E *}$ from the NRTL model under different MEA concentrations and temperatures. The calculated $\Delta F^{E *}$ from measured density and viscosity is positive while the excess viscosity $\eta^{E}$ calculated from (7) gives negative values for the low MEA concentration region indicating weak intermolecular attractions and gives positive values for high MEA concentration region signifying strong interactions.

$$
\eta^{E}=\eta-\sum_{i=1}^{n} x_{i} \eta_{i}
$$

( $\mathrm{n}=2$ for MEA $+\mathrm{H}_{2} \mathrm{O}$ mixtures and $\mathrm{n}=3$ for $\mathrm{AMP}+$ $\mathrm{MEA}+\mathrm{H}_{2} \mathrm{O}$ mixtures)

The ratio of $\Delta G^{E *} / \Delta F^{E *}$ was determined and following correlations is proposed with $R^{2}=0.99$.

$$
\begin{aligned}
& -\Delta G^{E *} / \Delta F^{E *}=f\left(x_{M E A}, T\right) \\
& f\left(x_{M E A}, T\right)=a+b x_{M E A} T+c T^{2}
\end{aligned}
$$

The suggested correlation was used to replace $\Delta F^{E *}$ in (3) and the viscosities were obtained accordingly. Figure 2 illustrates the comparison between measured viscosity and the correlation fit for aqueous MEA. The fit was in good agreement with measured data with AARD of $1.3 \%$ and AMD (maximum deviation) of 1.0 $\mathrm{mPa} \cdot \mathrm{s}$ as given in Table 4. This deviation is acceptable for engineering calculations and can be used to develop correlations for the $\mathrm{CO}_{2}$ loaded solutions. The estimated parameters for the correlation shown in (9) are given in Table 2.

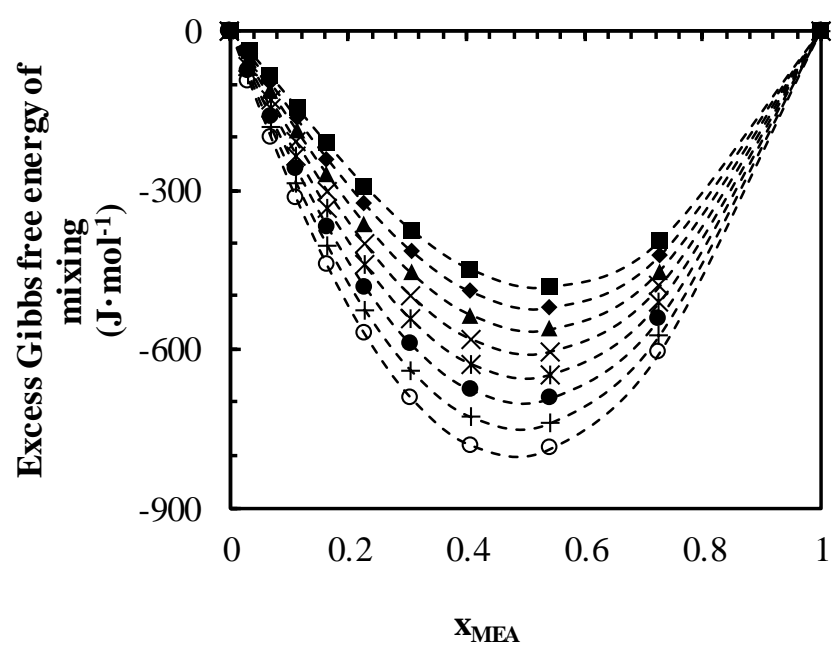

Figure 1. The variation of excess Gibbs free energy vs MEA mole fraction and temperatures: $293.15 \mathrm{~K}$, ' $\circ$ ';

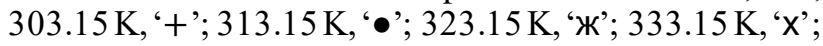
$343.15 \mathrm{~K}$, ' $\boldsymbol{\Delta}$ '; $353.15 \mathrm{~K}$, ' $\diamond ; 363.15 \mathrm{~K}$, ' $\boldsymbol{~}$ '.

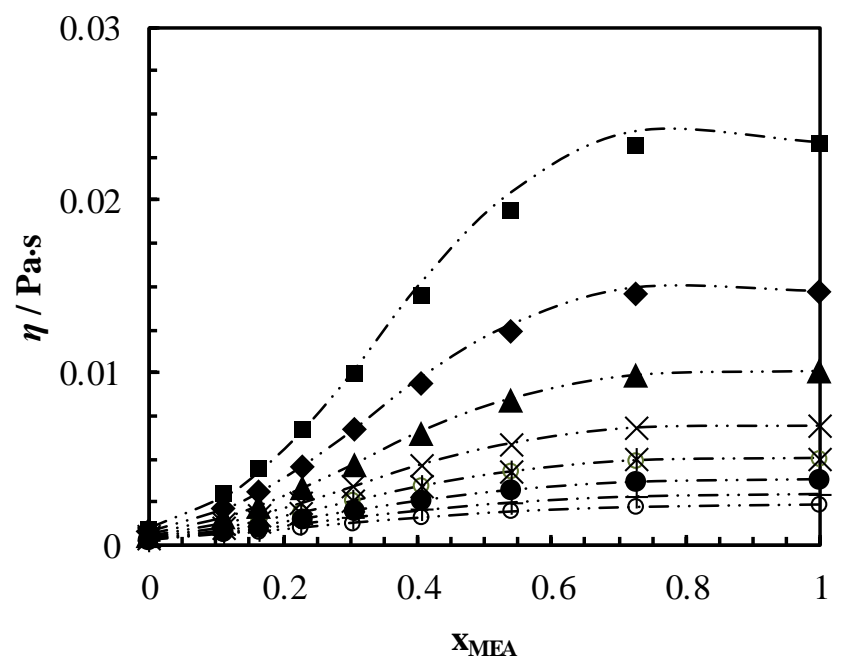

Figure 2. Comparison of measured viscosity of MEA + $\mathrm{H}_{2} \mathrm{O}$ mixtures with correlation at temperatures: $293.15 \mathrm{~K}$,

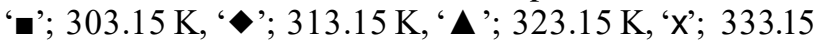

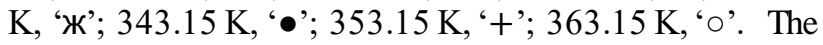
dash - dotted lines represent the correlation. 
Table 2. Estimated Parameters for Correlation of Viscosity of Aqueous MEA.

\begin{tabular}{|c|c|c|l|}
\hline $\begin{array}{c}\text { MEA } \\
(w t \%)\end{array}$ & $\begin{array}{c}\text { Temperature } \\
(K)\end{array}$ & $\begin{array}{c}\text { No. } \\
\text { points }\end{array}$ & Parameters \\
\hline $0-100$ & $293.15-363.15$ & 72 & $\begin{array}{l}\mathrm{a}=0.2801 \pm 0.008 \\
\mathrm{~b}=(5.557 \pm 0.0 .164) \times 10^{-04} \\
\mathrm{c}=(-1.623 \pm 0.0735) \times 10^{-06}\end{array}$ \\
\hline
\end{tabular}

Table 3. The Estimated Binary Parameters for the Correlation Shown in (10-13).

\begin{tabular}{|c|c|c|c|c|}
\hline \multicolumn{2}{|c|}{ Parameter } & $A M P+M E A$ & $M E A+\mathrm{H}_{2} \mathrm{O}$ & $\mathrm{AMP}+\mathrm{H}_{2} \mathrm{O}$ \\
\hline \multirow{3}{*}{$A_{0}$} & $a_{00}$ & $-1.724 \times 10^{4}$ & 141.854 & -117.059 \\
\cline { 2 - 5 } & $a_{01}$ & -9.370 & 0.562 & 0.296 \\
\cline { 2 - 5 } & $a_{02}$ & -2.516 & 0.598 & 0.623 \\
\hline \multirow{3}{*}{$A_{1}$} & $a_{10}$ & $-1.870 \times 10^{5}$ & -143.070 & 141.824 \\
\cline { 2 - 5 } & $a_{11}$ & -97.727 & -0.992 & -0.040 \\
\cline { 2 - 5 } & $a_{12}$ & 101.381 & 0.540 & 0.609 \\
\hline \multirow{2}{*}{$A_{2}$} & $a_{20}$ & $5.812 \times 10^{6}$ & 111.435 & -119.768 \\
\cline { 2 - 5 } & $a_{21}$ & $5.348 \times 10^{3}$ & 0.473 & 0.558 \\
\cline { 2 - 5 } & $a_{22}$ & $-2.233 \times 10^{3}$ & -0.168 & -0.067 \\
\hline
\end{tabular}

The $\Delta G^{E *}$ for AMP + MEA $+\mathrm{H}_{2} \mathrm{O}$ mixtures were examined using the NRTL model. Figure 3 shows the calculated $\Delta G^{E *}$ for the mixtures considered in this work. The $\Delta G^{E *}$ is negative for the considered AMP concentrations and temperatures. Further, negative $\eta^{E}$ implies weak intermolecular interactions for the range of AMP concentrations and temperatures. As discussed in the MEA $+\mathrm{H}_{2} \mathrm{O}$ mixtures, the ratio (r) of $-\Delta G^{E *} / \Delta F^{E *}$ was determined and a correlation was proposed as given in (10-13) to find the best fit for AMP $+\mathrm{MEA}+\mathrm{H}_{2} \mathrm{O}$ mixtures.

$$
\begin{aligned}
& -\Delta G^{E *} / \Delta F^{E *}=f\left(x_{A M P}, x_{M E A}, x_{H_{2} O}, T\right) \\
& \text { The ratio }-\Delta G^{E *} / \Delta F^{E *}=r_{12}+r_{23}+r_{13} \\
& r_{j k}=x_{j} x_{k} \sum_{i=0}^{n} A_{i}\left(x_{j}-x_{k}\right)^{i} \\
& A_{i}=a_{i o}+a_{i 1}(T)+a_{i 2}(T)^{2}
\end{aligned}
$$

The proposed correlation was able to represent measured viscosities with acceptable accuracy as illustrated by AARD and AMD in Table 4. Figure 4 shows the comparison of the correlation with measured data in which maximum deviations were observed at low temperatures. These deviations are smaller compared to the MEA $+\mathrm{H}_{2} \mathrm{O}$ mixtures since only three different compositions were considered for the study.

Table 4. Calculated AARD\% and AMD (mPa.s) for Comparison of Correlation with Measured Data.

\begin{tabular}{|l|c|c|}
\hline \multicolumn{1}{|c|}{ Mixture } & AARD (\%) & $\begin{array}{c}A M D \\
(\text { mPa.s })\end{array}$ \\
\hline $\mathrm{MEA}+\mathrm{H}_{2} \mathrm{O}$ & 1.3 & 1.0 \\
\hline $\mathrm{AMP}+\mathrm{MEA}+\mathrm{H}_{2} \mathrm{O}$ & 0.3 & 0.02 \\
\hline
\end{tabular}

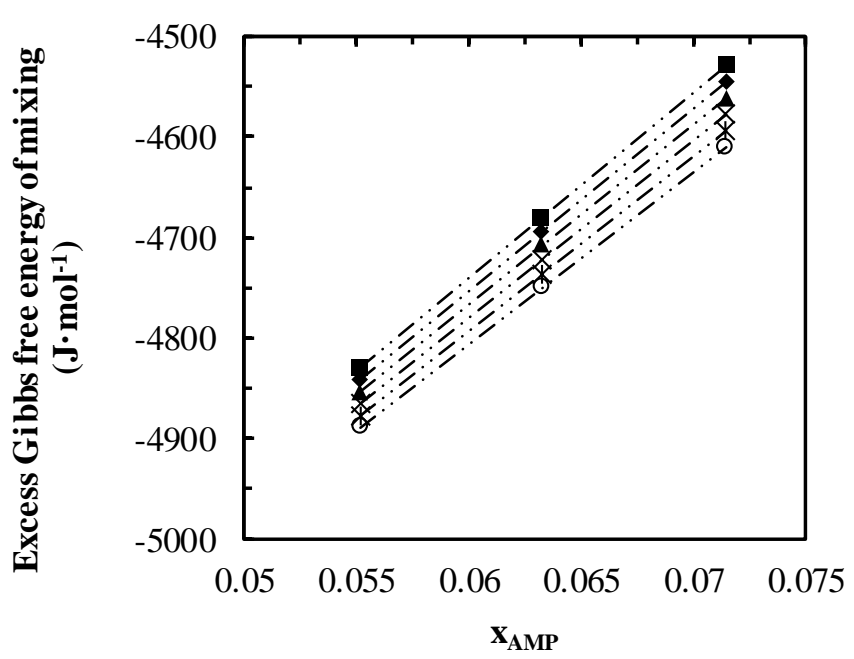

Figure 3. The variation of excess Gibbs free energy vs AMP mole fractions and temperatures: $293.15 \mathrm{~K}$, 'O';

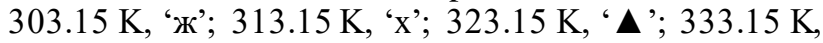
' '; $343.15 \mathrm{~K}$, ' $\mathbf{}$

The viscosity of $\mathrm{CO}_{2}$ loaded AMP $+\mathrm{MEA}+\mathrm{H}_{2} \mathrm{O}$ mixtures are highly important in the design and mathematical modelling and simulations of $\mathrm{CO}_{2}$ capture process based on absorption. The correlation discussed in this study for AMP + MEA $+\mathrm{H}_{2} \mathrm{O}$ mixtures can be adopted to developed viscosity correlations for $\mathrm{CO}_{2}$ loaded solutions using measured data. For use in e.g. a process simulation program like Aspen HYSYS or Aspen Plus, It is shown that the viscosities can be estimated by Hartono's correlation (Hartono et al., 2014) with fitted parameters for MEA $+\mathrm{H}_{2} \mathrm{O}$ mixtures with AARD $4.2 \%$ and the semiempirical model discussed in this work can estimate viscosity with $1.3 \%$ AARD. Mandal et al., (2003) used the Grunberg and 
Nissan correlation (Li and Lie, 1994) to fit the viscosity data with $3.08 \%$ AARD and it is higher than that from this study for AMP + MEA $+\mathrm{H}_{2} \mathrm{O}$ mixtures.

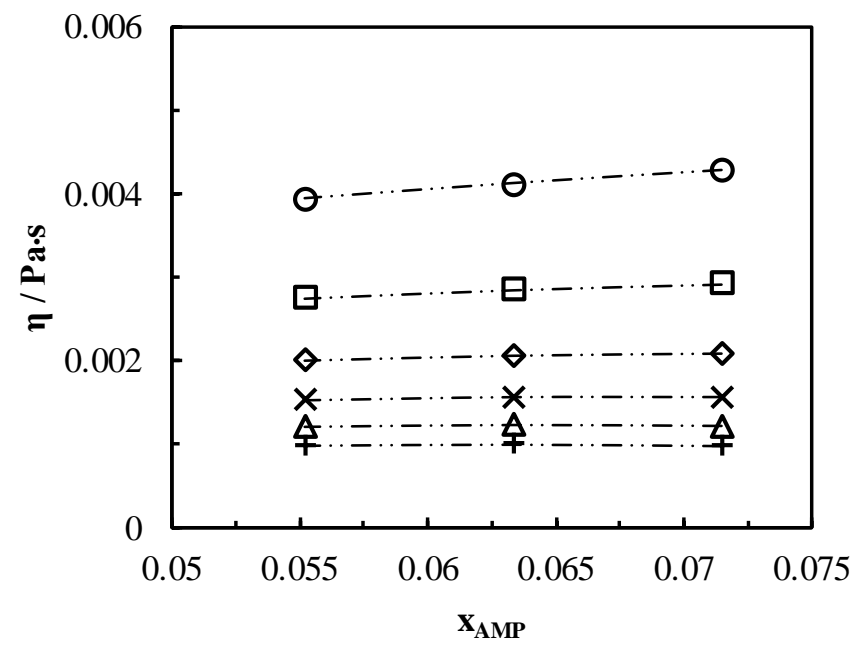

Figure 4. Comparison of measured viscosity of AMP + MEA $+\mathrm{H}_{2} \mathrm{O}$ mixtures with correlation at temperatures:

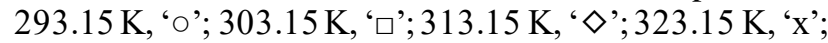
$333.15 \mathrm{~K}$, ' $\triangle$ '; $343.15 \mathrm{~K}$, '+'. The dash - dotted lines represent the correlation.

\section{Conclusion}

This work presents the applicability of the NRTL model to represent viscosities of MEA $+\mathrm{H}_{2} \mathrm{O}$ and AMP + MEA $+\mathrm{H}_{2} \mathrm{O}$ mixtures. The Eyring's viscosity model was adopted to determine excess free energy of activation for viscous flow. Correlations based on the regression for the ratio between excess Gibbs free energy of mixing from NRTL model and excess free energy of activation for viscous flow was proposed to represent measured viscosities. The accuracy of the correlation predictions are acceptable as the AARD (\%) is 1.3 and 0.3 for MEA $+\mathrm{H}_{2} \mathrm{O}$ and AMP $+\mathrm{MEA}+\mathrm{H}_{2} \mathrm{O}$ mixtures respectively. The NRTL model is available in the Aspen Plus commercial software to determine vapor - liquid equilibrium. In this paper, it is shown that these types of correlations can be integrated to determine viscosity in aqueous alkanolamines.

\section{References}

T. G. Amundsen, L. E. Øi, and D. A. Eimer. Density and viscosity of monoethanolamine+water+carbon dioxide from (25 to 80) ${ }^{\circ}$ C. J. Chem. Eng. Data, 54: 3096-3100, 2009.

R. B. Bird, W. E. Stewart, and E. N. Lightfoot. Transport Phenomena (second edition). USA: John Wiley \& Sons Inc., 2002.

W. Cao, K. Knudsen, A. Fredenslund, and P. Rasmussen. Group-contribution viscosity predictions of liquid mixtures using UNIFAC-VLE parameters. Ind. Eng. Chem. Res, 32: 2088-2092, 1993.
H. Eyring. Viscosity, Plasticity, and Diffusion as example of absolute reaction rates. Journal of chemical physics, 4: 283291, 1936.

A. Hartono, M. O. Mba, and H. F. Svendsen. Physical properties of partially $\mathrm{CO}_{2}$ loaded aqueous monoethanolamine (MEA). J. Chem. Eng. Data, 59: 18081816,2014

A. Hartono, M. Saeed, A. F. Ciftja, and H. F. Svendsen. Modeling of binary and ternary VLE of the AMP/Pz/ $\mathrm{H}_{2} \mathrm{O}$ system. Energy Procedia, 37: 1736-1743, 2013.

Y.-F. Hu. Prediction of viscosity of mixing electrolyte solutions based on the Eyring's absolute rate theory and the equations of Patwardhan and Kumar. Chemical Engineering Science, 59: 2457-2464, 2004.

M. N. Islam, M. M. Islam, and M. N. Yeasmin. Viscosity of aqueous solution of 2-methoxyethanol, 2-ethoxyethanol, and ethanolamine. J. Chem. Thermodynamics, 36:889-893, 2004.

M. J. Lee and T. K. Lin. Density and viscosity for Monoethanolamine+Water,+Ethanol, and +2 -Propanol. $J$. Chem. Eng. Data, 40: 336-339, 1995.

M.-H. Li and Y.-C. Lie. Densities and viscosities of solutions of Monoethanolamine $+\mathrm{N}$-Methyldiethanolamine + water and Monoethanolamine + 2-Amino-2-methyl-1-propanol + water. J. Chem. Eng. Data, 39: 444-447, 1994.

B. P. Mandal, M. Kundu, and S. S. Bandyopadhyay. Density and viscosity of aqueous solution of $(\mathrm{N}$ Methyldiethanolamine + Monoethanolamine $),(\mathrm{N}-$ Methyldiethanolamine + Diethanolamine), (2-Amino-2methyl-1-propanol + Monoethanolamine), and (2-Amino2-methyl-1-propanol + Diethanolamine). J. Chem. Eng. Data, 48: 703-707, 2003.

R. J. Martins, M. J. D. M. Cardoso, and O. E. Barcia. Excess Gibbs free energy model for calculating the viscosity of binary liquid mixtures. Ind. Eng. Chem. Res, 39: 849-854, 2000.

N. S. Matin, J. E. Remias, and K. Liu. Application of electrolyte-NRTL model for prediction of the viscosity of carbon dioxide loaded aqueous amine solutions. Ind. Eng. Chem. Res, 52: 16979-16984, 2013.

R. A. McAllister. The viscosity of liquid mixtures. A.I.Ch.E. Journal, 6: 427-431, 1960.

L. T. Novak, C.-C. Chen, and Y. Song. Segment-Based Eyring-NRTL viscosity model for mixtures containing polymers. Ind. Eng. Chem. Res, 43: 6231-6237, 2004.

J. M. Prausnitz, R. N. Lichtenthaler, and E. G. d. Azevedo. Molecular thermodynamics of fluid-phase equilibria (Third Edition). Prentice Hall PTR, 1999.

O. Redlich and A. T. Kister. Algebraic representation of thermodynamic properties and the classification of solutions. Ind. Eng. Chem., 40(2): 345-348, 1948.

K. A. G. Schmidt, Y. Maham, and A. E. Mather. Use of the NRTL equation for simultaneous correlation of vapourliquid equilibria and excess enthalpy. Journal of Thermal Analysis and Calorimetry, 89: 61-72, 2007.

G. M. Wilson. Vapor-Liquid Equilibrium. XI. A New Expression for the Excess Free Energy of Mixing. J. Am. Chem. Soc, 86: 127-130, 1964. 